Hope College

Hope College Digital Commons

Faculty Publications

8-23-2019

\title{
Functional and Anatomical Variations in Retinorecipient Brain Areas in Arvicanthis niloticus and Rattus norvegicus: Implications for the Circadian and Masking Systems
}

\author{
Dorela D. Shuboni-Mulligan \\ Michigan State University \\ Breyanna L. Cavanaugh \\ Michigan State University \\ Anne Tonson \\ Michigan State University \\ Erik M. Shapiro \\ Michigan State University \\ Andrew J. Gall \\ Hope College, gall@hope.edu \\ Follow this and additional works at: https://digitalcommons.hope.edu/faculty_publications \\ Part of the Behavioral Neurobiology Commons, and the Biological Psychology Commons
}

\section{Recommended Citation}

Repository citation: Shuboni-Mulligan, Dorela D.; Cavanaugh, Breyanna L.; Tonson, Anne; Shapiro, Erik M.; and Gall, Andrew J., "Functional and Anatomical Variations in Retinorecipient Brain Areas in Arvicanthis niloticus and Rattus norvegicus: Implications for the Circadian and Masking Systems" (2019). Faculty Publications. Paper 1494.

https://digitalcommons.hope.edu/faculty_publications/1494

Published in: Chronobiology International, Volume 36, Issue 11, August 23, 2019, pages 1464-1481.

Copyright @ 2019 Taylor \& Francis.

This Article is brought to you for free and open access by Hope College Digital Commons. It has been accepted for inclusion in Faculty Publications by an authorized administrator of Hope College Digital Commons. For more information, please contact digitalcommons@hope.edu. 
Functional and anatomical variations in retinorecipient brain areas in Arvicanthis niloticus and Rattus norvegicus: Implications for the circadian and masking systems

Dorela D. Shuboni-Mulligan ${ }^{\mathrm{a}}$, Breyanna L. Cavanaugh ${ }^{\mathrm{b}}$, Anne Tonson $^{\mathrm{c}}$, Erik M. Shapiro ${ }^{\mathrm{a}}$, and Andrew J. Gall ${ }^{\mathrm{d}^{*}}$

${ }^{a}$ Department of Radiology, Michigan State University, East Lansing, MI; ${ }^{b}$ Neuroscience Program, Michigan State University, East Lansing, MI; 'Department of Physiology, Michigan State University, East Lansing, MI; ${ }^{d}$ Department of Psychology, Hope College, Holland, MI

*corresponding author

Andrew J. Gall, Ph.D.

Department of Psychology

Hope College

35 E. 12 Street

Holland, MI, USA, 49424

Phone: 616.395.7729

Fax: 616.395.7163

e-mail: gall@hope.edu 


\title{
Functional and anatomical variations in retinorecipient brain areas in Arvicanthis niloticus and Rattus norvegicus: Implications for the circadian and masking systems
}

\author{
Daily rhythms in light exposure influence the expression of behaviour by \\ entraining circadian rhythms and through its acute effects on behaviour (i.e., \\ masking). Importantly, these effects of light are dependent on the temporal niche \\ of the organism; for diurnal organisms, light increases activity, whereas for \\ nocturnal organisms, the opposite is true. Here we examined the functional and \\ morphological differences between diurnal and nocturnal rodents in \\ retinorecipient brain regions using Nile grass rats (Arvicanthis niloticus) and \\ Sprague-Dawley (SD) rats (Rattus norvegicus), respectively. We established the \\ presence of circadian rhythmicity in cFOS activation in retinorecipient brain \\ regions in nocturnal and diurnal rodents housed in constant dark conditions to \\ highlight different patterns between the temporal niches. We then assessed \\ masking effects by comparing cFOS activation in constant darkness (DD) to that
} in a 12:12 light/dark (LD) cycle, confirming light responsiveness of these regions during times when masking occurs in nature. The intergeniculate leaflet (IGL) and olivary pretectal nucleus (OPN) exhibited significant variation among time points in DD of both species, but their expression profiles were not identical, as SD rats had very low expression levels for most timepoints. Light presentation in LD conditions induced clear rhythms in the IGL of SD rats but eliminated them in grass rats. Additionally, grass rats were the only species to demonstrate daily rhythms in LD for the habenula and showed a strong response to light in the superior colliculus. Structurally, we also analysed the volumes of the visual brain regions using anatomical MRI, and we observed a significant increase in the relative size of several visual regions within diurnal grass rats, including the lateral geniculate nucleus, superior colliculus, and optic tract. Altogether, our results suggest that diurnal grass rats devote greater proportions of brain volume to visual regions than nocturnal rodents, and cFOS activation in these brain regions is dependent on temporal niche and lighting conditions.

Keywords: diurnality, visual system, circadian, light exposure, magnetic resonance imaging 


\section{Introduction}

Temporal niche refers to the time-of-day during the light-dark cycle in which an organism is active and is defined by the unique levels of ambient light and temperature of the environment present (Kronfeld-Schor \& Dayan, 2008). These variations in ambient light and temperature can produce a range of activity patterns, with the two extremes restricting activity primarily to either the day (diurnal; Fogo et al., 2018) or night (nocturnal; Refinetti, 2006). Circadian rhythms and acute responses to light (i.e., masking) have uniquely adapted to regulate activity to these two temporal niches. Mechanisms underlying temporal niche preference are hypothesized to lie downstream of or independent from the suprachiasmatic nucleus ( $\mathrm{SCN}$, mammalian biological clock), as rhythms within the SCN are virtually identical in diurnal and nocturnal species (Smale, Nunez \& Schwartz, 2008), even though behaviours occur at opposite times of day. With respect to masking patterns, light increases activity in diurnal species, whereas light suppresses activity in nocturnal species (Shuboni et al., 2012). Light is one of the most important and powerful entraining stimuli (i.e., Zeitgeber), and in mammals, light information is solely communicated to the brain via the visual system (Ibuka, Inouye, \& Kawamura, 1977; Nelson \& Zucker, 1981). Therefore, it is important that we understand how light affects the brain of both diurnal and nocturnal organisms.

Structurally, the visual systems of diurnal and nocturnal animals have evolved differently due to evolutionary pressure to accommodate the challenges of each temporal niche (Ankel-Simons \& Rasmussen, 2008). As compared to diurnal animals, nocturnal animals have developed larger eyes (Garamszegi et al., 2001; Kirk, 2006), their retinas contain fewer cone receptors and more rod receptors (Ahnelt and Kolb, 2000; Peichl et al., 2000; Peichl, 2005; Solovei et al., 2009), and their optic nerves are significantly smaller (Stephan et al., 1984; Kirk \& Kay, 2004). All of these characteristics allow nocturnal animals to adapt adequate vision for environments with 
low light levels. Diurnal animals, on the other hand, have evolved vision for high light levels and have retinas rich in cone receptors (Gaillard et al., 2008; Peichl, 2005). In the Nile grass rat (Arvicanthis niloticus), a rodent model that is predominantly diurnal in the field and in the laboratory (Blanchong et al., 1999; Blanchong \& Smale, 2000), the retina contains ten times the number of cones compared to nocturnal mice and rats (Gaillard et al., 2008) and electroretinograms show several visual acuity features that more closely resemble human retinal physiology (Gilmour et al., 2008). Grass rats also differentiate from their nocturnal counterparts in the projections of intrinsically photosensitive retinal ganglion cells (ipRGC), the photoreceptor responsible for circadian entrainment and masking. Specifically, as compared to nocturnal rodents, grass rats exhibit significantly less innervation from ipRGCs in the lateral geniculate nucleus and the olivary pretectal nucleus, two brain regions critical for vision (Langel et al., 2015). Grass rats, therefore, are an ideal model to study the relationship between the visual system and both circadian rhythms and masking.

Grass rats are a murid rodent indigenous to the grasslands of Kenya (Delany \& Monro, 1986). In the laboratory, they are predominantly diurnal as measured by general activity patterns and body temperature (Katona \& Smale, 1997; McElhinny, Smale, \& Holekamp, 1997) and are considered a reliable diurnal rodent model (Refinetti, 2006) under these conditions; significant variations in chronotype only exist when grass rats are given a running wheel (Blanchong et al., 1999). Circadian and daily rhythms in cFOS activation within grass rat brain have been compared to nocturnal counterparts in many subregions, including within the circadian system (Nunez et al., 1999; Mahoney, Bult \& Smale, 2001; Schwartz, Nunez \& Smale, 2004), regions associated with sleep and wakefulness (Martínez, Smale \& Nunez, 2002; Novak, Smale \& Nunez, 2000; Nixon \& Smale, 2004; Schwartz \& Smale, 2005) and regions associated with reward 
(Castillo-Ruiz et al., 2010). However, these studies have not examined all retinorecipient regions, and it has been recently shown that some of these brain regions exhibit distinct neuronal activation in response to light that are unique from nocturnal rodents (Gall et al., 2014; Langel et al., 2014; Shuboni et al., 2015). Previous work has brought attention to two structures, the geniculate complex and the olivary pretectal nucleus, as possible components of the masking neural mechanism. Lesions of both areas alter the behaviours of the grass rat, both in masking response to light and circadian/daily rhythms in activity (Gall et al., 2013, 2014, 2017). We do not, however, yet know the circadian patterns of neuronal activity within these regions nor how daily light exposure masks these rhythms under normal lighting conditions. Many of these retinorecipient brain regions have been shown to contribute to circadian rhythm regulation in nocturnal species (reviewed in Morin \& Allen, 2006), but we do not yet know the pattern of expression in diurnal species, such as grass rats, or how FOS expression changes when animals are exposed to light during the day. Examining how light affects these brain regions differently in diurnal and nocturnal rodents under normal lighting conditions will allow us to better understand the different mechanisms by which these brain regions strengthen daily rhythms in behavior between temporal niches.

Here we examined the functional and structural differences of the visual system between the diurnal grass rat and the nocturnal Sprague Dawley rat. The expression of the immediate early protein, $\mathrm{cFOS}$, was used to examine neuronal activation within visual regions of the brain. Rhythms in cFOS expression under constant darkness provided insight into circadian rhythmicity within the brain regions. We then compared these rhythms to $12: 12$ light/dark conditions to measure the impact of light on the rhythmic expression of neuronal activation across the day and directly at the two 
timepoints where light is presented, which highlighted how nocturnal and diurnal rodents mask to the presentation of light under natural daily light exposure. Finally, to understand the structural differences of the visual system between temporal niches, we used high resolution magnetic resonance imaging (MRI) to measure the threedimensional volumes of visual regions and compared the differences in relative size. Both the functional and anatomical studies demonstrated the differences between temporal niches.

\section{Methods}

\section{Animals}

All experiments were approved by the Institutional Animal Care and Use Committee (IACUC) at Michigan State University and are in accordance with the NIH Guide for the Care and Use of Laboratory Animals. A total of seventy-six adult male diurnal grass rats (breeding colony, Michigan State University) and sixty-three adult male nocturnal SD rats (Charles River Laboratories, Wilmington, MA) were used in the study. All animals were housed in standard 12:12 light/dark conditions prior to experimentation and were provided food and water ad libitum throughout the duration of the study. Two experiments were conducted to compare the visual systems of the diurnal grass rat and nocturnal SD rat. In Experiment 1, we examined the circadian rhythms of brain activation, using cFOS, within several retinorecipient brain regions from slides produced for a previous publication (Schwartz et al., 2004). Tissue was collected from both species at six timepoints (Zeitgeber time (ZT)1, 5, 13, 17, 20, and 23; grass rat: $\mathrm{n}=35$; SD rat: $\mathrm{n}=29$ ) in 12:12 light/dark (LD) conditions and at six timepoints in constant darkness (DD; Circadian time (CT)1, 5, 13, 17, 20, and 23; grass rat: n=35; SD rat: $n=30$ ). For the 65 animals sacrificed in $\mathrm{DD}$, they were initially housed in 12:12 LD 
using cage-top infrared motion detectors to detect general activity patterns for 1-2 weeks, and then they were placed in DD for 21-22 days for grass rats, or 16-17 days for SD rats. At the end of this time frame in DD, activity data were visualized using actograms, and onsets were eye-fitted independently by two investigators. Perfusion times were randomly assigned, with CT0 indicating activity onset for grass rats, and CT12 indicating activity onset for SD rats (see Schwartz et al., 2004). In Experiment 2, we examined the total volume of several retinorecipient brain areas using ex vivo highresolution magnetic resonance imaging (MRI; grass rat: n=6; SD rat: n=4; see Ex vivo MRI Protocol below for details). All animals in Experiment 2 were males, singlyhoused, and between 6 months and 1 year old when perfused. All perfusions for Experiment 2 occurred during the lights-on phase, between ZT4 and ZT8.

\section{Immunohistological Procedure}

Tissue was collected and stained as previously described in Schwartz et al. (2004). Briefly, animals were transcardially perfused using 0.01 M PBS followed by $4 \%$ paraformaldehyde. Brains were removed and post-fixed for 1-2 $\mathrm{h}$ and then transferred to a $20 \%$ sucrose solution for at least $48 \mathrm{~h}$ until sectioning. Coronal sections were cut on a freezing microtome at $30 \mu \mathrm{m}$ and stored in cryoprotectant at $-20{ }^{\circ} \mathrm{C}$ until further processing.

Free floating sections were rinsed three times in PBS for 10 min then blocked for $1 \mathrm{~h}$ in 5\% normal goat serum (Vector Laboratories, Burlingame, CA). Tissue was incubated in the primary antibody, rabbit anti-cFOS (1:25,000; Santa Cruz Biochemistry, Santa Cruz, CA), for $48 \mathrm{~h}$ at $4{ }^{\circ} \mathrm{C}$ then moved into the secondary antibody, biotinylated goat anti-rabbit (1:200; Vector Laboratories), for $1 \mathrm{~h}$ at room temperature. An avidin-biotin peroxidase complex kit (ABC Vectastain Kit; Vector 
Laboratories) was used before visualizing the protein with $0.5 \mathrm{mg} / \mathrm{ml}$ diaminobenzidine (DAB; Sigma, St. Louis, MO). Sections were washed three times with PBS and then mounted on gelatinized slides. Slides were dehydrated with increasing concentrations of alcohol and xylenes, then coverslipped with Permount (Fisher Scientific, Hampton, $\mathrm{NH})$.

\section{cFOS rhythms in the Visual System}

Images were acquired with a Zeiss light microscope (Zeiss, Gottingen, Germany) equipped with a digital camera (CX900, MBF Bioscience, Williston, VT). All images were combined into one composition file using Adobe Photoshop 7 (Adobe Systems, Mountain View, CA). Regions of interest were identified within the images, either by using counting boxes (superior colliculus (SC), $300 \mu \mathrm{m} \times 400 \mu \mathrm{m}$ ) or were outlined by a trained researcher blind to condition; olivary pretectal nucleus (OPN), lateral habenula (LHb), medial habenula (MHb), dorsal lateral geniculate nucleus (dLGN), intergeniculate nucleus (IGL), and ventral lateral geniculate nucleus (vLGN) (see Figure 1 for outlined brain areas). The number of cells positive for cFOS (cFOS+) was counted bilaterally for one section using the Particle Analysis tool and thresholding in the ImageJ Program (NIH, Bethesda, MD). Thresholding was performed by converting the photomicrograph into a monochrome image, and the threshold was set manually using the slider bars until the maximum number of cells containing cFOS were pixelated. Finally, the number of cFOS+ cells were counted automatically using ImageJ.

\section{Ex vivo MRI Protocol}

Perfused whole brains were transferred into a $15 \mathrm{~mL}$ tube filled with Fombilin ${ }^{\circledR} \mathrm{Y}$ (Sigma-Aldrich, St Louis, MO), a perfluorocarbon solution that produces no MRI signal and is the "gold standard" for ex vivo imaging biological samples. Tubes were secured 
to a $2 \times 2$ surface receive array within a volume transmit coil. Images were acquired with a 7T Bruker Biospec 70/30 USR using a 2D T2-weighted TurboRARE sequence (TE:33 ms, TR:2654.1 ms, Rare Factor:8, $50 \mu \mathrm{m} \times 50 \mu \mathrm{m})$ for $1 \mathrm{~h} 1 \mathrm{~min}(30$ repetitions) with 10 slices for grass rats and 12 slices ( $1 \mathrm{~mm}$ thickness) for SD rats.

One brain from each species was soaked for $48 \mathrm{hr}$ in a PBS solution doped with 0.1M gadopentetate dimeglumine (Gd-DTPA, Magnevist $\left.{ }^{\circledR}\right)$. These brains were transferred, prior to scanning, to fresh PBS in a $15 \mathrm{~mL}$ tube which was cut to fit into a home-made $17 \mathrm{~mm}$ solenoid coil. Images were also acquired with a 7T Bruker Biospec 70/30 USR using 3D T1-/T2-weighted Flash sequence (TE:10.44 ms, TR:31 ms, 100 $\mu \mathrm{m} \times 100 \mu \mathrm{m} \times 100 \mu \mathrm{m})$ for $47 \min (2$ repetitions).

\section{Volume Analysis of Visual System}

Volume analysis for the high resolution $\mathrm{T}_{2}$-weighted images was conducted using the Measure tool in the ImageJ Program (NIH, Bethesda, MD). Three components of the visual system were clearly visible with MRI: SC, lateral geniculate nucleus (LGN), Habenula (EPI), and optic tract (opt). Two non-visual areas were also outlined as controls, the cortex (CTX) and hippocampus (HPC). The whole brain was then outlined to correct for total volume differences between the two species. A percentage was calculated by dividing the volume of each area by the whole brain volume and then multiplying by 100 . Prior to statistical analysis, percentage data was arcsine transformed. The 3D MRI were used to create 3D volume rendering of the regions of interest using 3D Slicer 4.8 (https://www.slicer.org/; Fedorov et al., 2012).

\section{Statistical Analyses}

The histological data was compared separately for lighting condition (LD or DD) and for species (grass rats or SD rats) using one-way ANOVAs with time of day as the 
independent variable and the number of cFOS+ cells as the dependent variable. For each region of interest, the number of cFOS+ cells were compared across time followed by post hoc analysis using t-tests (Tukey HSD). The presence of 24-h rhythms was detected using cosinor analysis, with data represented by the following function: $x i=M$ $+A * \cos [(2 * \mathrm{pi} * \mathrm{ZT}) / 24]$, where M denotes MESOR and A denotes amplitude of the oscillation. Linear regression by method of least squares was used to test for rhythmicity, and the probability that $\mathrm{A}$ is significantly different from zero was calculated using an F-test with 2 and $N-3$ degrees of freedom (Cornelissen, 2014; Nelson et al., 1979; Tong, 1976; Refinetti, Lissen, \& Halberg, 2007). Additionally, within species $\mathrm{CT}$ and ZT were compared at two timepoints, 1 and 5, to examine the direct effects of light on cFOS using a two-way ANOVA. For the MRI volume data, independent samples t-tests were used to compare the size of each region between grass rats and SD rats. All analyses were performed with SPSS Statistic 23 software (IBM Corp., Armonk, NY) and significance for all tests was $p<0.05$.

\section{Results}

\section{Circadian Rhythms of cFOS in Constant Dark Conditions}

We examined significant variation among time points of neuronal activity within retinorecipient regions using cFOS activation across time in constant dark conditions, including the geniculate complex (e.g., dLGN, IGL, vLGN), OPN, habenula (e.g., MHb and $\mathrm{LHb}$ ), and SC. Figure 1 presents photomicrographs of cFOS in the geniculate complex, OPN, and habenula (EPI); photomicrographs of cFOS in the SC can be found in Supplemental Figure 1. Grass rats exhibited significant variation among time points in cFOS protein expression within the OPN, $\mathrm{F}(5,31)=3.840, p=0.011$, with post-hocs revealing higher levels of activation at CT5 and CT23 (Figure 2, left panel). When the 
OPN was analyzed using cosinor analyses, however, a significant circadian rhythm was not detected (Supplemental Table 2). Within the geniculate complex, grass rats had two regions that trended toward significance from the ANOVA; the IGL, F(5,31)=2.604, $p=0.051$, and the vLGN, $\mathrm{F}(5,31)=2.364, p=0.068$. The IGL $\mathrm{cFOS}$ expression was highest at CT17 and CT20 during the beginning of subjective night. Of these two brain regions, only the IGL exhibited a significant circadian rhythm following cosinor analyses (Supplemental Table 2). cFOS activation for the dLGN did not express a significant variation among time points from the ANOVA $(\mathrm{F}(5,32)=1.209, p=0.421)$ or a circadian rhythm from cosinor analyses. Neither the $\operatorname{SC}(\mathrm{F}(5,31)=0.604, p=0.697)$ nor either portion of the grass rat habenula $(\mathrm{LHb}, \mathrm{F}(5,31)=0.789, p=0.566 ; \mathrm{MHb}$, $\mathrm{F}(5,31)=1.904, p=0.126)$ exhibited a significant variation among time points in $\mathrm{cFOS}$ activation from the ANOVA. Cosinor analyses supported these findings, as a significant circadian rhythm was not detected in the SC or habenula of grass rats.

In the SD rats, there was a significant effect of time on cFOS activation in the IGL, $\mathrm{F}(5,28)=3.023, p=0.031$, however the pattern of activation was low and exhibited the lowest cFOS expression at CT20 (Figure 2, right panel), and was not significant following cosinor analyses. The OPN of the SD rat also expressed a significant variation among time points in constant conditions $(\mathrm{F}(5,27)=3.807, p=0.012)$, but again the levels of cFOS were low with only CT17 having a heightened level of activation. Again, cosinor analyses did not reveal a significant circadian rhythm in the OPN (Supplemental Table 2). No rhythms were expressed within the dLGN $(F(5,28)=1.221$, $p=0.331), \operatorname{vLGN}(\mathrm{F}(5,28)=2.104, p=0.101), \mathrm{SC}(\mathrm{F}(5,28)=1.106, p=0.392), \mathrm{LHb}$ $((\mathrm{F}(5,28)=0.991, p=0.445)$, or $\mathrm{MHb}(\mathrm{F}(5,28)=0.855, p=0.526)$, according to the ANOVA or cosinor analyses (Supplemental Table 2). 


\section{Daily Rhythms of cFOS in 12:12 Light/Dark Conditions}

We examined the daily rhythms of neuronal activity within same retinorecipient regions using cFOS activation across time in a 12:12 light/dark cycle (Figure 3 for geniculate complex, OPN, \& EPI; Supplemental Figure 1 for SC). Remarkably, in the grass rat, all regions that exhibited significant circadian rhythms in cFOS expression in constant darkness became arrhythmic in 12:12 LD conditions (IGL, $F(5,33)=0.711$, $\mathrm{p}=0.621$; vLGN, F(5,33)=0.563, p=0.727; OPN, F(5,34)=1.542, p=0.210; Figure 4, left panel), because cFOS activation was heightened in the light (see Figure 5). However, both regions of the habenula expressed significant variation among time points in cFOS expression in $\mathrm{LD}$ conditions $(\mathrm{LHb}, \mathrm{F}(5,33)=4.633, \mathrm{p}=0.003 ; \mathrm{MHb}, \mathrm{F}(5,34)=3.018$, $\mathrm{p}=0.026$ ), and cosinor analyses revealed that these variations fit a sinusoidal wave. The $\mathrm{SC}(\mathrm{F}(5,33)=1.702, \mathrm{p}=0.167)$ remained arrhythmic as it was in DD.

In SD rats, two regions of the geniculate complex expressed significant variations among time points in cFOS activity in 12:12 LD conditions: the dLGN $(\mathrm{F}(5,29)=4.409, p=0.005)$ and the IGL $(\mathrm{F}(5,29)=9.974, p<0.001)$. However, cFOS expression patterns were different between these two regions. In the IGL, post-hocs revealed that levels were significantly higher during the active period and the beginning of rest period, while in the dLGN, two peaks occurred at ZT1 and ZT17. Cosinor analyses revealed significant rhythms only in the IGL (Supplemental Table 2). The pattern of expression within the vLGN, which was not significant $F(5,29)=1.212$, $p=0.334$, more closely resembled the dLGN. The OPN of the SD rat did not exhibit a significant daily rhythm in cFOS activation, $\mathrm{F}(5,29)=1.008, p=0.436$ (Figure 4 , right panel). No other regions expressed significant daily rhythms in cFOS activation (SC, $\mathrm{F}(5,29)=1.860, p=0.143 ; \mathrm{LHb}, \mathrm{F}(5,29)=0.548, p=0.738 ; \mathrm{MHb}, \mathrm{F}(5,29)=0.254, p=0.934)$.

We compared cFOS levels between ZT and CT groups at the two timepoints 
during the day, ZT/CT1 \& 5, to examine the direct effect of light on the cFOS expression (Figure 5 and Supplemental Table 1). In grass rats, light during the day in ZT conditions increased cFOS levels with main effects of lighting condition for the SC $(\mathrm{F}(1,20)=17.61, p<0.001), \mathrm{dLGN}(\mathrm{F}(1,20)=6.46, p=0.019)$ and $\mathrm{a}$ trend toward significance in the IGL $(\mathrm{F}(1,20)=4.22, p=0.053)$. The OPN had a significant interaction $(\mathrm{F}(1,20)=7.673, p=0.012)$, when the time points were compared between CT and ZT there was a 5.6x increase in expression one hour after lights on in ZT conditions when compared to $\mathrm{CT}, \mathrm{t}(10)=2.621, p=0.026$. SD rats had a similar directional pattern with dramatic increases in cFOS expression with main effects of light exposure in three regions: $\operatorname{dLGN}(\mathrm{F}(1,16)=10.84, p=0.005)$, $\operatorname{IGL}(\mathrm{F}(1,16)=54.57, p<0.001)$ and $\mathrm{OPN}$ $(\mathrm{F}(1,15)=17.23, p=0.001)$. The OPN had a $5.2 \mathrm{X}$ increase in expression at ZT1 and a 7.9X increase at ZT5. The SC for the SD rat did not have significant increase in cFOS expression as a ME of lighting $\mathrm{F}(1,15)=0.09, p=0.770)$ or an interaction $(\mathrm{F}(1,15)=2.27$, $\mathrm{p}=0.153$ ). Altogether, grass rats exhibited significant increases in light-induced FOS in the dLGN, IGL, OPN, and SC at ZT1, whereas SD rats exhibited significant increases at the same time point in only the dLGN, IGL, and OPN. We observed similar effects at ZT5, except for the OPN, which no longer exhibited a significant increase in lightinduced FOS in grass rats. These results demonstrate that grass rats and SD rats express differential activation to the presentation of light within our nuclei of interest.

\section{MRI Volume Analysis}

We assessed the high resolution 2D images taken across the brains of grass rat and SD rat at 7 levels (Figure 6). The four components of the visual system (SC, LGN, opt, and EPI) are highlighted in representative sections (Figure 7). Clear size differences between the two species are visible in overall size and proportion of most of the visual 
areas. Quantification of total brain volume of grass rats $(526.2 \pm 6.7 \mathrm{~mm} 3)$ showed a significantly smaller brain than SD rats $(1198.7 \pm 13.7 \mathrm{~mm} 3 ; \mathrm{t}(8)=48.672, \mathrm{p}<0.001)$, with a $56.1 \%$ decrease in total volume. Both control regions assessed did not differ in total percent volume between grass rats and SD rats, $\mathrm{HPC}(\mathrm{t}(8)=0.162, \mathrm{p}=0.875)$ and $\mathrm{CTX}$ $(\mathrm{t}(8)=1.087, \mathrm{p}=0.309)$. There were significant differences in percent volume of 3 visual regions examined, $\operatorname{LGN}(\mathrm{t}(8)=12.503, \mathrm{p}<0.001), \mathrm{SC}(\mathrm{t}(8)=5.610, \mathrm{p}<0.001)$ and opt $(\mathrm{t}(8)=7.411, \mathrm{p}<0.001)$, but not in the EPI $(\mathrm{t}(8)=1.163, \mathrm{p}=0.278$; Figure 7$)$. Within the LGN, SC, and opt grass rats had larger percent volume when compared to SD rats, with an increase of $47.0 \%, 47.4 \%, 48.8 \%$ respectively. When these regions were visualized in 3D MRI scans, the dramatic increase in size within the grass rats when compared with the SD rat can be clearly observed from coronal, sagittal and horizontal sections (Figure 8).

\section{Discussion}

Circadian rhythms and masking work synergistically in the presence of light (12:12 LD cycle) to define the daily expression of activity rhythms (Aschoff, 1999). There are clear differences within the neural mechanisms that drive these behaviours between species that occupy different temporal niches (Yan, Smale, \& Nunez, 2018). The present experiments demonstrate the differences within the visual system between diurnal and nocturnal rodent species in both (1) circadian and masking responses in neuronal activation and (2) the morphology of the structural components.

The IGL has been shown to play a crucial role in defining masking behaviour between the temporal niches (Gall et al., 2013). Specifically, in grass rats, lesions of the IGL alter the direction of masking behaviour in response to the presentation of light from positive to negative, therefore causing the diurnal rodent to respond to light similar 
to a nocturnal species by suppressing activity. Understanding the activation patterns based on time of day will provide insight into how the region works to promote diurnal behaviour through masking and circadian rhythms in natural 12:12 LD conditions by demonstrating the natural activity in DD and how light alters responses in LD. We found here that while grass rats showed a trend toward significant variation among time points of cFOS activation in the IGL under constant dark conditions and significant circadian rhythms following cosinor analyses, SD rats did not. For grass rats, higher activation was observed during the night (CT17 \& CT20). The magnitude of cFOS activation was generally low in SD rats and did not have a clear rhythmic pattern. Although cFOS expression in the IGL was low overall, cosinor analyses did detect a rhythm in grass rats. In addition, these results are similar to the number of light induced FOS cells in the IGL of grass rats in previous reports (Shuboni et al., 2015). In 12:12 LD conditions, we found no daily rhythm in cFOS expression broadly within the IGL for grass rats, whereas SD rats did exhibit a significant rhythm in LD. Increased cFOS activation specifically during the light phase (Figure 5) masked the rhythm that is normally present in constant darkness. For grass rats, this is similar to previous findings that reported no significant effect of time when comparing the daily rhythms in the region at ZT4 and ZT16 (Smale et al., 2001). However, these authors found a difference in cFOS within NPY+ cells, which project to the SCN, between the day and night, suggesting a conduit for masking effects that merits further examination. In nocturnal rodents, similar patterns of IGL activation have been observed. Edelstein et al. (2000) showed that Wistar rats exhibited markedly low levels of cFOS expression in the IGL under the DD conditions but had clear and significant rhythms in 12:12 LD cycle. These rhythms were attributed to light activation of neurons within the IGL, with low activation in constant darkness and high activation during both LL and the light portion 
of the 12:12 LD cycle. Light pulses at night have also been shown to induce increases of cFOS in the IGL in Fisher rats (Caldelas et al., 1998; Prichard et al., 2002) and blind mole-rats (Oelschlager et al., 2000). This increase in cFOS to a light pulse, however, was not observed in CD1 mice at ZT14 (Shuboni et al., 2015). Here we observed lower levels of cFOS during subjective day in constant dark conditions that were activated following the presentation of light in 12:12 LD cycles; this pattern of increased activation in both grass rats and SD rats during the light portion of the LD cycle was also observed in the OPN. Altogether, our results show that the IGL responds differently in 12:12 LD conditions in grass rats as compared to SD rats, suggesting that it plays an important role in temporal niche differences. Indeed, this is supported by lesion data of the IGL in diurnal and nocturnal species, which strongly suggests its involvement in temporal niche differences in masking behaviour (Gall et al., 2013; Redlin et al., 1999; Johnson et al., 1989; Pickard, 1989; Edelstein \& Amir, 1999).

The OPN has also been shown to play a major role in the masking behaviour of diurnal grass rats, eliminating the positive masking response to light and causing a positive masking response to darkness post-lesion (Gall et al., 2017). We report here that grass rats and SD rats expressed significant variation among time points in cFOS activation in the OPN under constant dark conditions, with heightened activation in the subjective night; however, in a LD cycle, daily rhythms were eliminated in both species. In both species, this elimination of rhythms was caused by an increase in the level of activation observed during the light phase. We have previously demonstrated that the OPN is responsive to the presentation of light at night in the grass rat (Shuboni et al., 2015) and that when masking behaviour in grass rats was inverted to a nocturnal phenotype with IGL lesioned animals, the OPN's responsiveness to light was no longer present (Gall et al., 2014). For the SD rat, the OPN had relatively low expression levels 
across DD and were more dramatically increased in LD conditions. Other studies in nocturnal rodents, showed that light pulses induce an increase of cFOS in the OPN of albino Fisher F344 rats (Caldelas et al., 1998; Prichard et al., 2002) and blind mole-rats (Oelschlager et al., 2000). Altogether, these results suggest that light induces cFOS expression in both the IGL and OPN of grass rats and SD rats, but there are clear differences between the species.

To better examine the effects of light on cFOS in our data set we compared the two timepoints during light phase, ZT1 and ZT5, to the constant dark, CT1 and CT5. Grass rats have been shown to respond more dramatically to the presentation of light with increases in activity across the $24 \mathrm{hr}$ period, while mice only decrease activity at one time (CT14; Shuboni et al., 2012). Here we observed cFOS activation to light in all brain regions examined, except the habenula and vLGN. Interestingly, both components of the habenula in grass rats were the only regions examined to show a daily rhythm in cFOS expression in LD conditions. A study has recently shown a marked difference in the level of GABAergic cells in the LHb between mice and grass rats (Langel et al., 2018) suggesting a possible role for the nuclei in masking behaviour. Similar findings of light inducing the expression of cFOS were observed for SD rats in the entire geniculate complex (e.g., DLG, IGL, OPN), but not for the SC. Grass rats, in stark contrast, had a significant light-induced increase in cFOS activation in the SC. The SC has been shown to be responsive to brief flashes of light and to moving and stationary visual patterns (Craner et al., 1992; Montero and Jian, 1995), but it not yet known how the SC responds to sustained periods of light. Our data show that the SC is more lightresponsive in grass rats than in SD rats following prolonged light presentation, suggesting a potential important species-difference in light functionality in this brain region. The clear functional difference found within these regions may have led to 
structural adaptations based on temporal niche of the population.

It should be noted that one limitation of our study was only examining two time points during the day, and four time points at night. Because previous work found significant changes in the lower subparaventricular zone in grass rats as compared to SD rats (Nunez et al., 1999), we were more interested in the changes that occurred at night. A future study is needed to more thoroughly examine changes that happen throughout the day, particularly towards the end of the lights-on period.

Evolution between temporal niches has led to the adaptations of many components of the visual system (Ankel-Simons et al., 2008). Since grass rats are more functionally sensitive to the presentation of light, here we also examined the structural differences in the visual system between the niches using MRI. Within primates, there have been many studies comparing variation in the size of eye structures (Kirk, 2004; Kirk, 2006) and visual regions within the brain, particularly the cortex and geniculate (Heesy, Kamilar \& Willms, 2011). Studies of adaptation within the rodent visual system between temporal niche switches, have demonstrated alterations in visual cortex (Heimel, Van Hooser, \& Nelson, 2005; Campi \& Krubitzer, 2010; Campi et al., 2011). We were particularly keen to examine differences between the niches in regions that were associated with masking behaviour. This study confirms that there is alteration in the relative size of optic tract, superior colliculus and geniculate complex between diurnal grass rats and nocturnal SD rats, with larger structures found in grass rats. These findings are in line with a previous histological study in grass rats which showed a larger SC, dLGN, and vLGN (Gaillard et al., 2013). Importantly, we further demonstrate that the input from the optic tract is also larger in grass rats, suggesting that for the diurnal grass rat these regions, (1) receive more visual information from the retina via the optic tract and (2) the devote a greater proportion of the brain to regions 
that are critical for driving diurnal behaviour. These anatomical results support our cFOS data and suggest that visual input into the non-image forming retinorecipient brain regions in grass rats plays an important role in promoting diurnal behaviour. This study is also the first to use high resolution MRI to compare the anatomical size of visual system structures between species of different temporal niches. The technique is a powerful tool for measuring in three-dimensions regions of interest within the brain without sacrificing tissue for histology.

It is important to note that there are many ways to assess rhythmicity in cFOS expression (Refinetti, Lissen, \& Halberg, 2007). The most common way that has been reported in multiple reports is using ANOVAs to detect significant variation among time points (Caldelas et al., 1998; Prichard et al., 2002). We also analyzed our data using cosinor analyses, which can be used to detect rhythms that fit a sinusoidal wave. We included both types of analyses here, and we note some similarities and differences between the ANOVAs and cosinor analyses. In constant conditions, the only rhythms detected using cosinor analyses were within the IGL in grass rats; none were significant in SD rats. In contrast, using ANOVAs, we detected significance in the IGL and OPN of both species. With respect to LD conditions, rhythms detected using cosinor analyses included only the IGL in SD rats and the habenula in grass rats; ANOVAs revealed significance in the IGL and dLGN of SD rats, along with the LHb and MHb of grass rats. Because rhythms do not always fit a sinusoidal wave, it is important to also include results from the ANOVA.

\section{Conclusion}

Grass rats are an optimal model for studying the relationship between the visual system and both circadian and masking mechanisms in a diurnal organism. Here we 
have used this model to examine circadian rhythms in neuronal activation within brain regions that receive direct ipRGC projections and how activation in these regions is altered in the presence of a light/dark cycle. Circadian rhythms of cFOS activation were observed in several retinorecipient brain areas in grass rats and SD rats housed in constant darkness (e.g., geniculate complex, OPN). The expression of cFOS activation in the geniculate complex and OPN was significantly higher at ZT1 and ZT5 as compared to CT1 and CT5, indicating light-induced cFOS expression in these brain areas. The masking response to light, which was defined here as FOS expression induced by a LD cycle that is above and beyond FOS expression in DD, also has similar effects in many regions when comparing between species, including the IGL, dLGN, and OPN, confirming the responsiveness of these regions during times when masking occurs in nature. Interestingly, whereas light-induced cFOS activation in the SC was observed in grass rats, it was not observed in SD rats, suggesting a species-specific difference in light responsiveness in this brain region. Importantly, whereas light increases behaviour in diurnal species such as grass rats, light suppresses behaviour in nocturnal species such as SD rats. Since most retinorecipient brain regions respond similarly in both species, we hypothesize that these brain regions must affect downstream circuitry differently, or that the differences in masking behaviour arises from different visual pathways.

Additionally, we use high resolution MRI for the first time to compare visual system morphology between temporal niches. These findings further confirm the adaptation of structure in the visual system in a diurnal rodent and mirror changes observed between diurnal and nocturnal organisms in avian and primate species (Schmitz \& Motani, 2010). We demonstrated that the optic nerve/tract, geniculate complex and the SC all are proportionally larger in grass rats which suggests a possible 
driver for the temporal niche specific heightened sensitivity to light in our diurnal species. In summary, we have demonstrated significant functional and morphological differences within the visual system between diurnal and nocturnal rodents. 


\section{Acknowledgements}

AJG was supported by a National Institutes of Health (NIH) Ruth L. Kirschstein National Research Service Award (NRSA) from the National Institute of Neurological Disorders and Stroke (NINDS) (F32 NS083360). We also thank the Hope College Psychology Department for their support of this project, and the Hope College Division of Social Sciences for providing startup funds to AJG. This work was also supported by a National Institutes of Mental Health grant (R01 MH53433) and a National Science Foundation grant (IOS 1051919). EMS and DDSM were supported by the National Cancer Institute grant (R21 CA185163). We would like to acknowledge Dr. Laura Smale for her input on experimental design and editing of the manuscript, and Dr. Tony Nunez for tissue collection.

\section{Disclosure of Interest}

The authors report no conflict of interest.

\section{Data Availability Statement}

The data that support the findings of this study are available from the corresponding author, [AJG], upon reasonable request. 


\section{References}

Ahnelt PK, Kolb H. 2000. The mammalian photoreceptor mosaic-adaptive design.

Progress in Retinal and Eye Research. 19(6): 711-777.

Ankel-Simons F, Rasmussen DT. 2008. Diurnality, nocturnality, and the evolution of primate visual systems. Am J Phys Anthropol. Suppl 47:100-117.

Aschoff J. 1999. Masking and parametric effects of high-frequency light-dark cycles. Jpn J Physiol. 49(1): 11-18.

Barker DJ, Sanabria F, Lasswell A, Thrailkill EA, Pawlak AP, Kileen PR. 2010. Brief light as a practical aversive stimulus for the albino rat. Behav Brain Res. 214(2): 402-408.

Blanchong JA, McElhinny TL, Mahoney MM, Smale L. 1999. Nocturnal and diurnal rhythms in the unstriped Nile rat, Arvicanthis niloticus. J Biol Rhythms. 14(5): 364.

Blanchong JA, Smale L. 2000. Temporal patterns of activity of the unstriped Nile rat, Arvicanthis niloticus. J Mammal. 81(2): 595-599.

Caldelas I, Salazar-Juarez A, Granados-Fuentes D, Escobar C, Aguilar-Roblero R. 1998. Circadian modulation of c-Fos expression occurs only in the SCN and not in other visual projection areas in the rat. Biological Rhythm Research. 29(5): 494-500.

Campbell BA, Messing RB. 1969. Aversion threshold and aversion difference limens for white light in albino and hooded rats. Journal of Experimental Psychology. 82(2):353-359.

Campi KL, Krubitzer L. 2010. Comparative studies of diurnal and nocturnal rodents: differences in lifestyle result in alterations in cortical field size and number. $\mathbf{J}$ Comp Neurol. 518(22): 4491-4512. 
Campi KL, Collins CE, Todd WD, Kaas J, Krubitzer L. 2011. Comparison of area 17 cellular composition in laboratory and wild-caught rats including diurnal and nocturnal species. Brain Behav Evol. 77:116-130.

Castillo-Ruiz A, Nixon JP, Smale L, Nunez AA. 2010. Neural activation in arousal and reward areas of the brain in day-active and night-active grass rats. Neuroscience. 165(2): 337-349.

Cha YJ, Lee JH, Baik TK, Park JS. 2011. C-Fos immunoreactivity in the neurons of the lateral geniculate nucleus in albino rats by light exposure after dark rearing. Korean J Ophthalmol. 25(6):434-439.

Cornelissen G. (2014). Cosinor-based rhythmometry. Theor Biol Med Model. 11: 16.

Craner SL, Hoffman GE, Lund JS, Humphrey AL, Lund RD. 1992. cFos labeling in rat superior colliculus: Activation by normal retinal pathways and pathways from intracranial retinal transplants. Experimental Neurology. 117 (3):219-229.

Delany MJ, Monro RH. 1986. Population dynamics of Arvicanthis niloticus (Rodentia: Muridae) in Kenya. J. Zool. Lond. (A). 209:85-103.

Edelstein K, Amir S. (1999). The role of the intergeniculate leaflet in entrainment of circadian rhythms to a skeleton photoperiod. J Neurosci. 19: 372-380.

Edelstein K, Beaule C, D’Abramo R, Amir S. 2000. Expression profiles of JunB and cFos proteins in the rat circadian system. Brain Research. 870:54-65.

Fedorov A, Beichel R, Kalpathy-Cramer J, Finet J, Fillion-Robin JC, Pujol S, Bauer C, Jennings D, Fennessy F, Sonka M, Buatti J, Aylward S, Miller JV, Pieper S, Kikinis R. 2012. 3D Slicer as an image computing platform for the Quantitative Imaging Network. Magnetic Resonance Imaging. 30(9): 1323-1341.

Fogo GM, Goodwin AM, Khacherian OS, Ledbetter BJ, Gall AJ. 2018. The effects of ambient temperature and lighting intensity on wheel-running behavior in a 
diurnal rodent, the Nile grass rat (Arvicanthis niloticus). Journal of Comparative Psychology. 133(2), 215-222.

Gaillard F, Bonfield S, Gilmour G S, Kuny S, Mema SC, Martin BT, Smale L, Crowder N, Stell WK, Sauvé Y. 2008. Retinal anatomy and visual performance in a diurnal cone-rich laboratory rodent, the Nile grass rat (Arvicanthis niloticus). Journal of Comparative Neurology. 510(5), 525-538.

Gaillard F, Karten HJ, Sauve Y. 2013. Retinorecipient areas in the diurnal murine rodent Arvicanthis niloticus: A disproportionally large superior colliculus. Journal of Comparative Neurology. 521(8), 1699-1726.

Gall AJ, Smale L, Yan L, Nunez AA. 2013. Lesions of the intergeniculate leaflet lead to a reorganization in circadian regulation and a reversal in masking responses to photic stimuli in the nile grass rat. PLoS ONE. 8(6): e67387.

Gall AJ, Yan L, Smale L, Nunez AA. 2014. Intergeniculate leaflet lesions result in differential activation of brain regions following the presentation of photic stimuli in Nile grass rats. Neuroscience Letters. 579:101-105.

Gall AJ, Khacherian OS, Ledbetter B, Deats SP, Luck M, Smale L, Yan L, Nunez AA. 2017. Normal behavioral responses to light and darkness and the pupillary light reflex are dependent upon the olivary pretectal nucleus in the diurnal nile grass rat. Neuroscience. 355: 255-237.

Garamszegi LZ, Moller AP, Erritzoe, J. 2001. Coevolving avian eye size and brain size in relation to prey capture and nocturnality. Proc R Soc Lond B. 269: 961-967.

Gilmour GS, Gaillard F, Watson J, Kuny S, Mema SC, Bonfield S, Stell WK, Sauvé Y. 2008. The electroretinogram (ERG) of a diurnal cone-rich laboratory rodent, the Nile grass rat (Arvicanthis niloticus). Vision Research. 48(27): 2723-2731. 
Heimel JA, Van Hooser SD, Nelson SB. 2005. Laminar organization of response properties in primary visual cortex of the gray squirrel (sciurus carolinensis). $\mathbf{J}$ Neurophysiol. 94: 3538-3554.

Heesy CP, Kamilar JM, Willms J. 2011. Retinogeniculate pathway components scale with orbit convergence only in primates and not other mammals. Brain Behav Evol. 77:105-115.

Ibuka N, Inouye ST, Kawamura H. 1977. Analysis of sleep-wakefulness rhythms in male rats after suprachiasmatic nucleus lesions and ocular enucleation, Brain Research. 122(1): 33-47.

Johnson RF, Moore RY, Morin LP. (1989). Lateral geniculate lesions alter circadian activity rhythms in the hamster. Brain Res Bull. 22: 411-422.

Katona C, Smale L. 1997. Wheel-Running Rhythms in Arvicanthis niloticus. Physiology \& Behavior. 61(3): 365-372.

Kirk EC. 2004. Comparative morphology of the eye in primates. The Anatomical Record Part A: Discoveries in Molecular, Cellular, and Evolutionary Biology banner. 281A(1):1095-1103.

Kirk CE. 2006. Effects of activity pattern on eye size and orbital aperture size in primates. Journal of Human Evolution. 51(2): 159-170.

Kirk EC, Kay RF. 2004. The evolution of high visual acuity in the anthropoidea. In: Ross C, Kay R, editors. Anthropoid origins: new visions. New York: Kluwer/Plenum. p $539-602$.

Kronfeld-Schor N, Dayan T. 2008. Activity patterns of rodents: the physiological ecology of biological rhythms. Biol Rhythm Res. 39(3), 193-211. 
Langel JL, Ikeno T, Yan L, Nunez AA, Smale, L. 2018. Distributions of GABAergic and glutamatergic neurons in the brains of a diurnal and nocturnal rodent. Brain Research. 1700: 152-159.

Langel JL, Smale L, Esquiva G, Hannibal J. 2015. Central melanopsin projections in the diurnal rodent, Arvicanthis niloticus. Frontiers in Neuroanatomy. 9, 93.

Langel J, Yan L, Nunez AA, Smale L. 2014. Behavioral masking and cfos response to light in day- and night-active grass rats. Journal of Biological Rhythms. 29(3): 192-202.

Lau C, Zhou IY, Cheung MM, Chan KC, Wu EX. 2011. BOLD Temporal Dynamics of rat superior colliculus and lateral geniculate nucleus following short duration visual stimulation. PLoS ONE. 6(4): e18914.

Mahoney M, Bult A, Smale L. 2001. Expression in the suprachiasmatic nucleus and adjacent hypothalamus of Arvicanthis niloticus. Journal of Biological Rhythms. 16(2):149-162.

Martínez GS, Smale L, Nunez AA. 2002. Diurnal and nocturnal rodents show rhythms in orexinergic neurons. Brain Research. 955(1-2): 1-7.

McElhinny TL, Smale L, Holekamp KE. 1997. Patterns of Body Temperature, Activity, and Reproductive Behavior in a Tropical Murid Rodent, Arvicanthis niloticus. Physiology \& Behavior. 62(1): 91-96.

Montero VM, Jian S. 1995. Induction of c-fos protein by patterned visual stimulation in central visual pathways of the rat. Brain Research. 690: 189-199.

Morin LP, Allen CN. 2006. The circadian visual system, 2005. Brain Res Rev. 51(1): 160.

Nelson W, Tong YL, Lee JK, Halberg F. 1979. Methods for cosinor-rhythmometry. Chronobiologia. 6(4): 305-323. 
Nelson RJ, Zucker I. 1981. Absence of extra-ocular photoreception in diurnal and nocturnal rodents exposed to direct sunlight. Comp. Biochem. Physiol. 69A: $145-148$.

Novak CM, Smale L, Nunez AA. 2000. Rhythms in Fos expression in brain areas related to the sleep-wake cycle in the diurnal Arvicanthis niloticus. Am J Physiol Regulatory Integrative Comp Physiol. 278:R1267-1274.

Nixon JP, Smale L. 2004. Individual differences in wheel-running rhythms are related to temporal and spatial patterns of activation of orexin A and B cells in a diurnal rodent (Arvicanthis niloticus). Neuroscience. 127:25-34.

Nunez AA, Bult A, McElhinny TL, Smale L. 1999. Daily rhythms of fos expression in hypothalamic targets of the suprachiasmatic nucleus in diurnal and nocturnal rodents. Journal of Biological Rhythms. 14(4): 300-306.

Oelschlager HHA, Nakamura M, Herzog M, Burda H. 2000. Visual system labeled by c-fos immunohistochemistry after light exposure in the 'blind' subterranean Zambian mole-rat (Cryptomys anselli). Brain, Behavior and Evolution. 55: 209220.

Peichl L. 2005. Diversity of mammalian photoreceptor properties: Adaptations to habitat and lifestyle? Anatomical Record Part a-Discoveries in Molecular Cellular and Evolutionary Biology. 287A(1), 1001-1012.

Peichl L, Kunzle H, Vogel P. 2000. Photoreceptor types and distributions in the retinae of insectivores. Visual Neuroscience. 17(6): 937-948.

Pickard GE. (1989). Entrainment of the circadian rhythm of wheel-running activity is phase shifted by ablation of the intergeniculate leaflet. Brain Res. 494: 151-154. 
Prichard JR, Stoffel RT, Quimby DL, Obermeyer WH, Benca RM, Behan M. 2002. FOS immunoreactivity in rat subcortical visual shell in response to illuminance changes. Neuroscience. 114(3): 781-793.

Redlin U, Vrang N, Mrosovsky N. (1999). Enhanced masking response to light in hamsters with IGL lesions. J Comp Physiol A. 184: 449-456.

Refinetti R. 2006. Variability of diurnality in laboratory rodents. J Comp Physiol A. 192: 701-714.

Refinetti R, Lissen GC, Halberg F. 2007. Procedures for numerical analysis of circadian rhythms. Biol Rhythm Res. 38(4): 275-325.

Schwartz MD, Nunez AA, Smale L. 2004. Differences in the suprachiasmatic nucleus and lower subparaventricular zone of diurnal and nocturnal rodents. Neuroscience 127(1):13-23.

Schwartz DM, Smale L. 2005. Individual Differences in Rhythms of Behavioral Sleep and Its Neural Substrates in Nile Grass Rats. Journal of Biological Rhythms. 20(6), 526-537.

Schmitz L, Motani R. 2010. Morphological differences between the eyeballs of nocturnal and diurnal amniotes revisited from optical perspectives of visual environments. Vision Research. 50(10):936-946.

Shuboni DD, Cramm S, Yan L, Nunez AA, Smale L. 2012. Acute behavioral responses to light and darkness in nocturnal Mus musculus and diurnal Arvicanthis niloticus. J Biol Rhythms. 27(4), 299-307. 
Shuboni DD, Cramm SL, Yan L, Ramanathan C, Cavanaugh BL, Nunez AA, Smale L. 2015. Acute effects of light on the brain and behavior of diurnal Arvicanthis niloticus and nocturnal Mus musculus. Physiology \& Behavior. 138: 75-86.

Smale L, McElhinny T, Nixon J, Gubik B, Rose S. 2001. Patterns of wheel running are related to fos expression in the neuropeptide-Y-Containing neurons in the Intergeniculate Leaflet. Journal of Biological Rhythms. 16(2):163-172.

Smale L, Nunez AA, Schwartz MD. 2008. Rhythms in a diurnal brain. Biol Rhythm Res. 39(3), 305-318.

Solovei I, Kreysing M, Lantot C, Kosem S, Peichl L, Cremer T, Guck J, Joffe B. 2009. Nuclear architecture of rod photoreceptor cells adapts to vision in mammalian evolution. Cell. 137(2): 356-368.

Stephan H, Frahm H, Baron G. 1984. Comparison of brain structure volumes in insectivora and primates. IV. Non-cortical visual structures. J Hirnforsch. 25(4), $385-403$.

Tong YL. 1976. Parameter estimation in studying circadian rhythms. Biometrics. 32(1), $85-94$.

Yan L, Smale L, Nunez A. 2018. Circadian and photic modulation of daily rhythms in diurnal mammals. European Journal of Neuroscience. Epub. 


\section{Figures}

\section{A. Grass Rat}
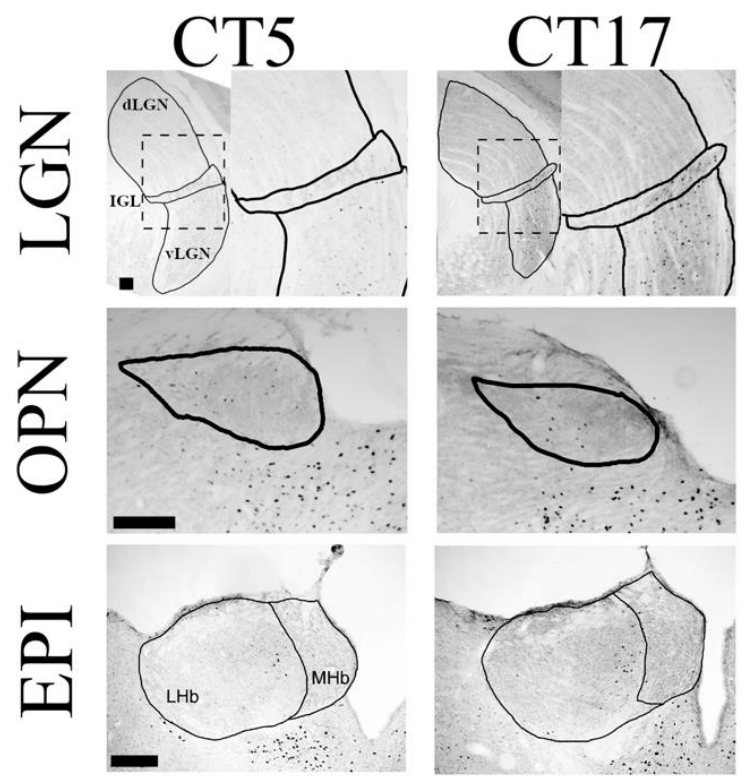

\section{B. SD Rat}

\section{CT5}
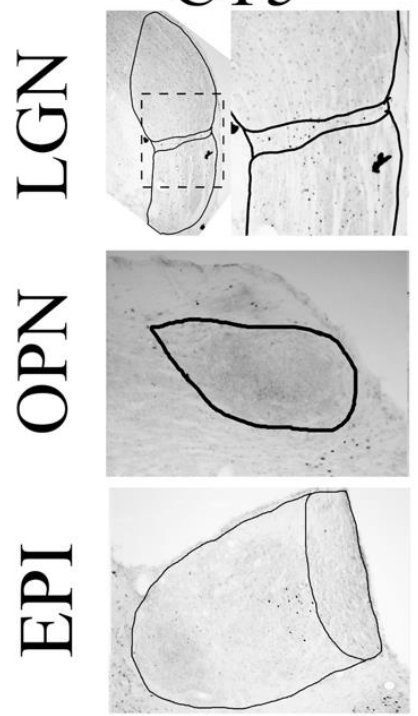

CT17
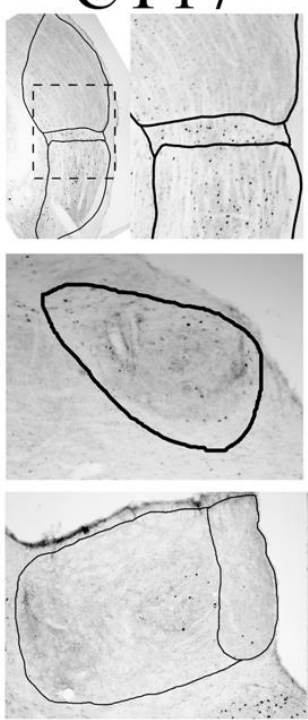

Figure 1. Photomicrographs of the cFOS expression at Circadian Time 5 and 17. In grass rats (A), photomicrographs of the lateral geniculate nucleus (LGN, top row), olivary pretectal nucleus (OPN, middle row), and habenula (EPI, lower row) show stained cFOS+ nuclei in constant dark conditions at Circadian Time (CT)5 and 17. In SD rats (B), photomicrographs of the LGN (top row), OPN (middle row) and habenula (lower row) also show cFOS+ nuclei in constant dark conditions at CT5 and 17. 
Grass Rat
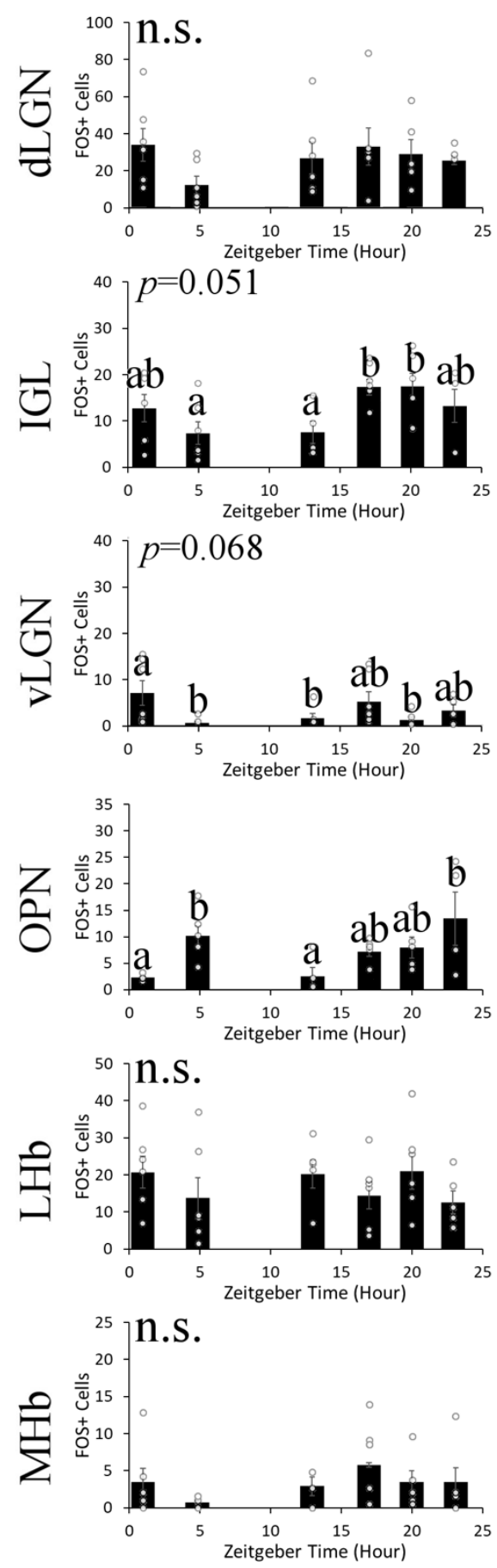

SD Rat
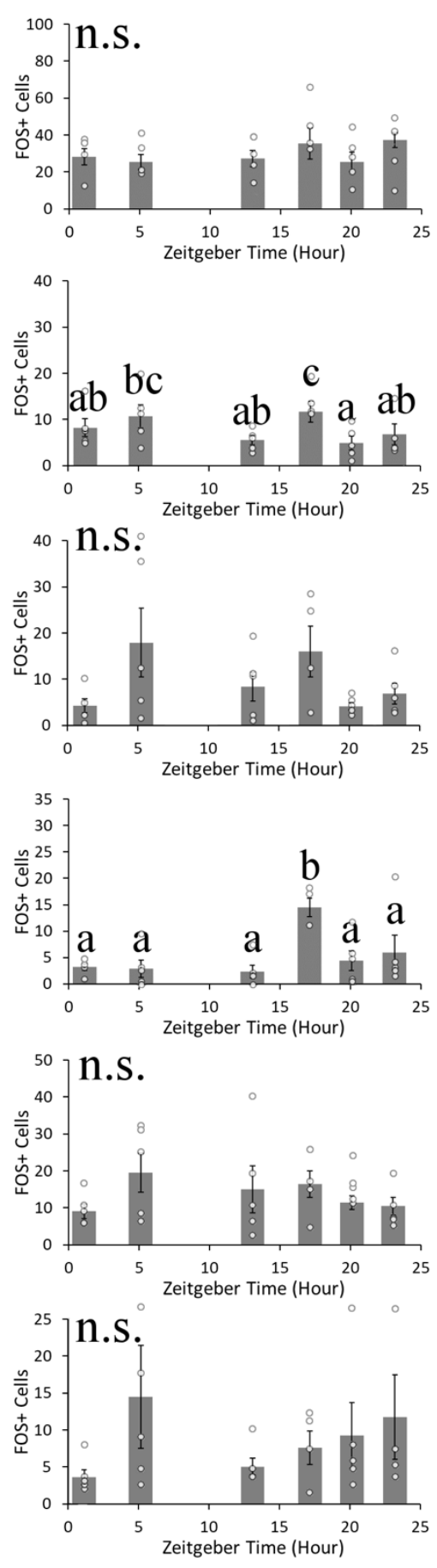

Figure 2.

Quantification of cFOS expression within retinorecipient regions across Circadian

Time. The total number of cFOS+ cells was compared across constant darkness for grass rats (left column, black bars) and SD rats (right, grey bars) in several visual structures. In the grass rats, the OPN exhibited significant differences across the day, and the IGL and vLGN were trending toward significance. SD rats had significant circadian rhythms of cFOS expression within the IGL and OPN. Different letters indicate significance with $\mathrm{p}<0.05$. 


\section{A. Grass Rat}

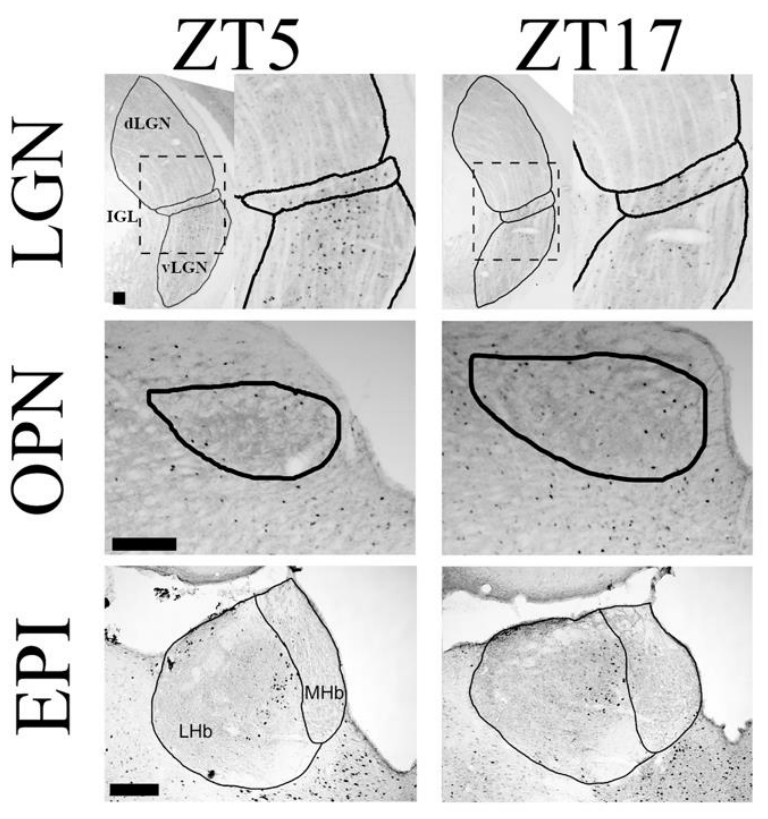

\section{B. SD Rat}
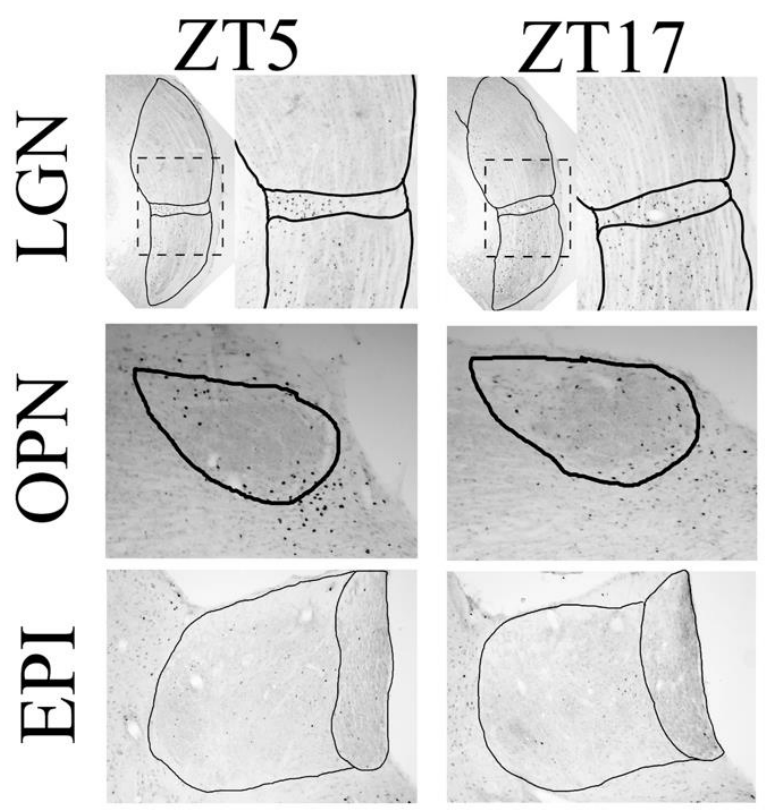

Figure 3. Photomicrographs of the cFOS expression at Zeitgeber Time 5 and 17. In grass rats (A), photomicrographs of the lateral geniculate nucleus (LGN, top row), olivary pretectal nucleus (OPN, middle row), and habenula (EPI, lower row) show stained cFOS+ cells in 12:12 LD conditions at Zeitgeber Time (ZT)5 and 17. In SD rats (B), photomicrographs of the LGN (top row), OPN (middle row) and habenula (lower row) also show cFOS+ nuclei in 12:12 LD conditions at ZT5 and 17. 
Grass Rat
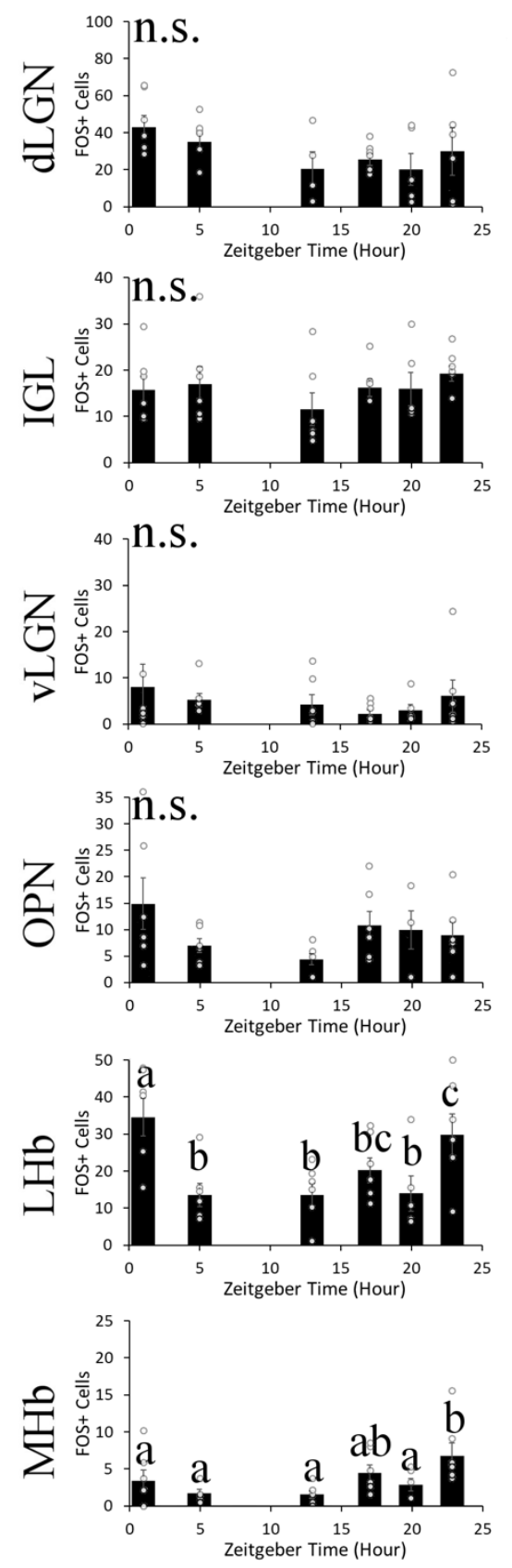

SD Rat
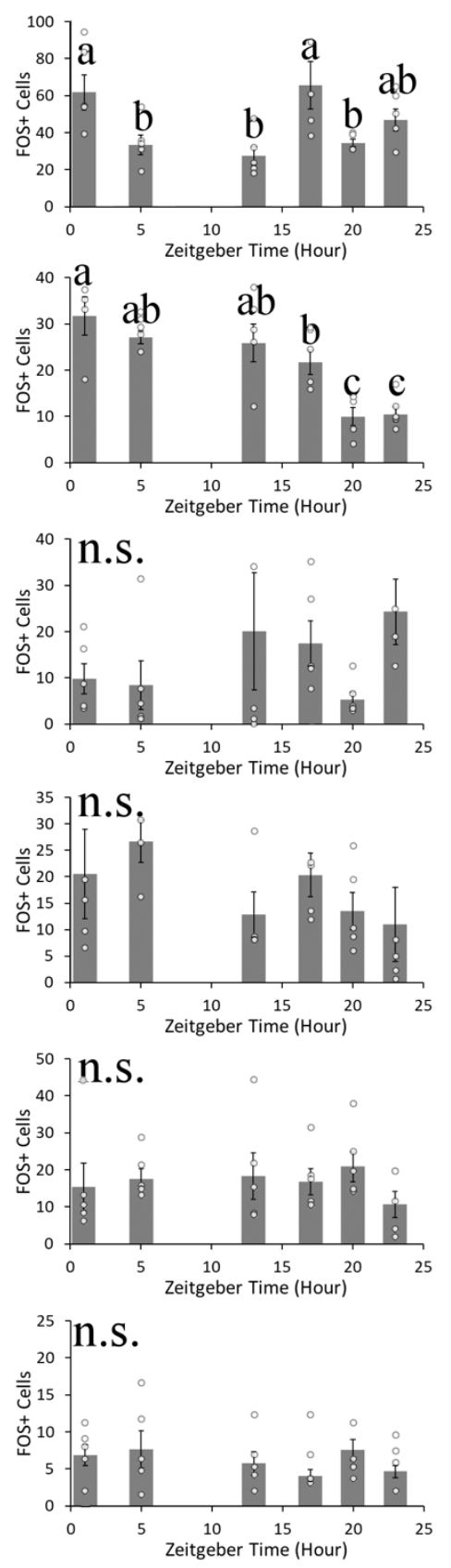

Figure 4. Quantification of cFOS expression within retinorecipient regions across Zeitgeber Time. The total number of cFOS+ cells was compared across 12:12 LD conditions for grass rats (left column, black bars) and SD rats (right, grey bars) in several visual structures. In the grass rats, the OPN and IGL no longer express significant daily rhythms, however both the LHb and MHb have daily rhythms under LD conditions. In contrast, SD rats exhibited significant circadian rhythms of cFOS expression within the dLGN and IGL. Different letters indicate significance with $\mathrm{p}<0.05$. 

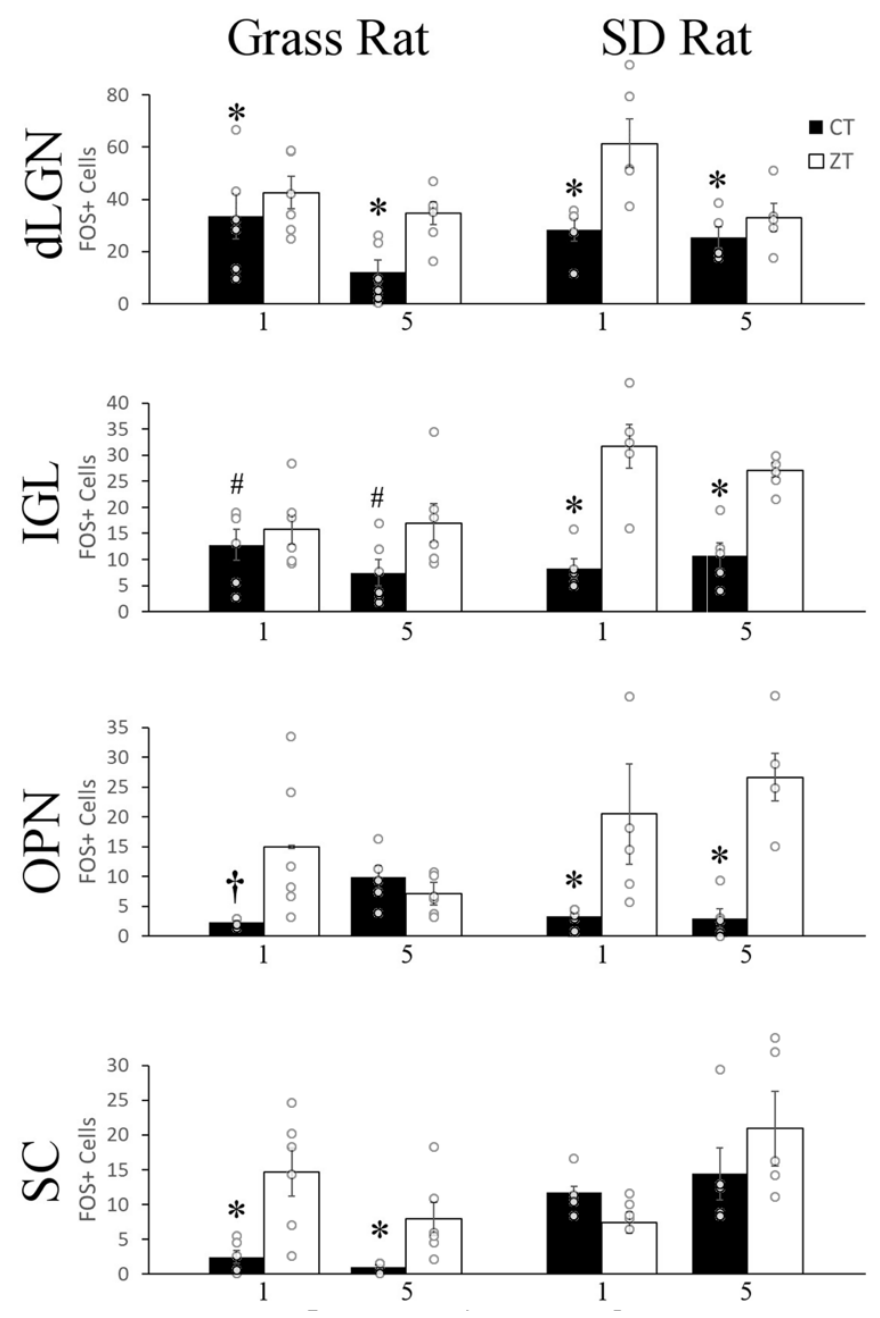

Figure 5. Quantification of masking effects of light between temporal niche. The total number of cFOS+ cells was compared for grass rats (left column) and SD rats (right column) in several visual structures at two timepoints in DD (black bars) and LD (white bars). Grass rats had a significant increase in cFOS+ cells during at least one time point for all regions depicted. SD rats had increases in expression during light for the dLGN, IGL, and OPN. * indicates a significant main effect of lighting with $\mathrm{p}<0.05$, \# indicates a main effect trend toward significance with $\mathrm{p}=0.053, \uparrow$ indicates a significant interaction with $\mathrm{p}<0.05$. 

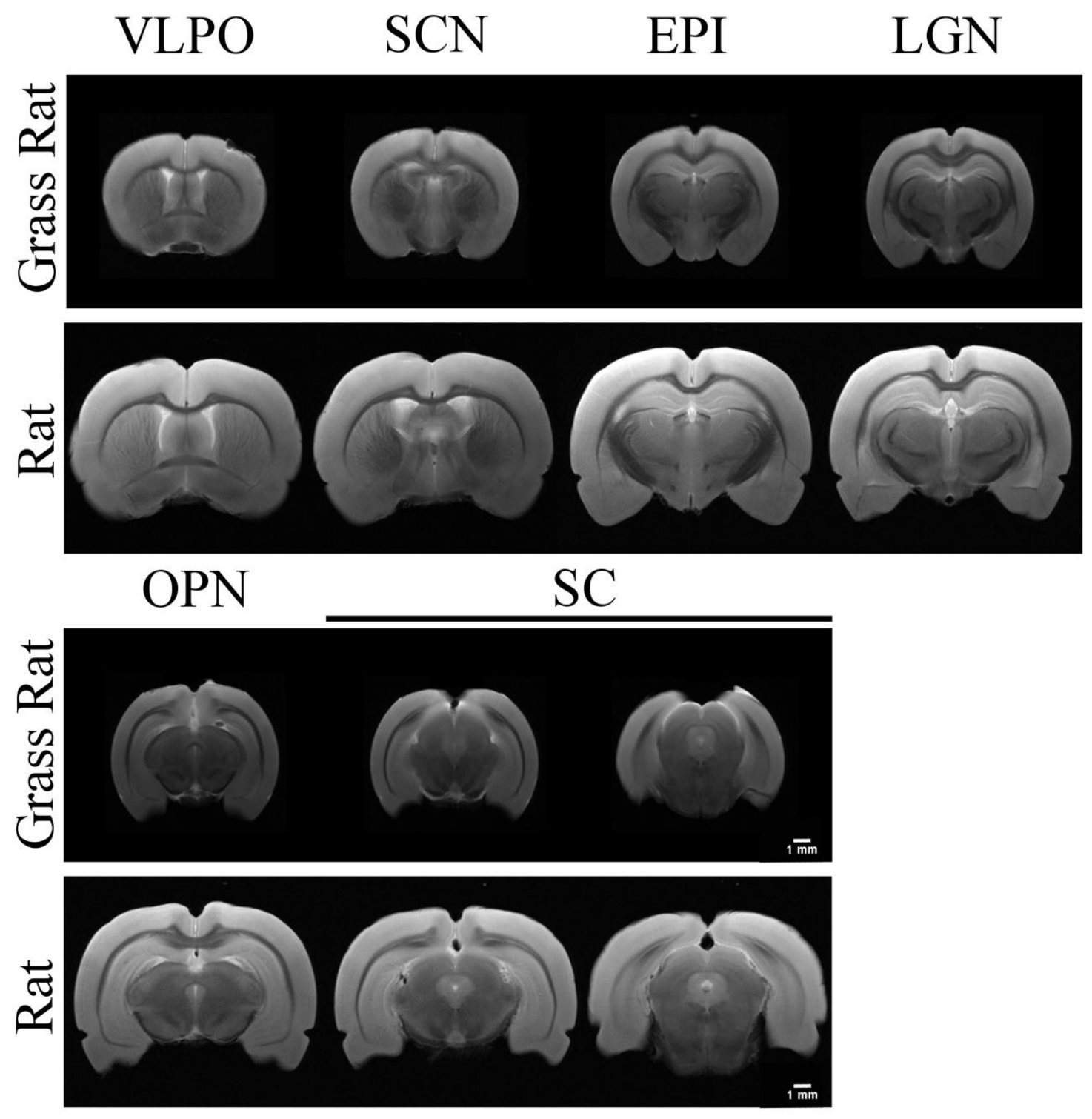

Figure 6. High resolution T2-weighted MRIs across both the grass rat and SD rat brains. Slices include sections at the levels of the VLPO, SCN, habenula (EPI), LGN, OPN, and the SC. Only some structures are clearly discriminated in the MRI and include the EPI, geniculate complex, and SC. Additionally, control regions the hippocampus and cortex are easily identified in both species. 

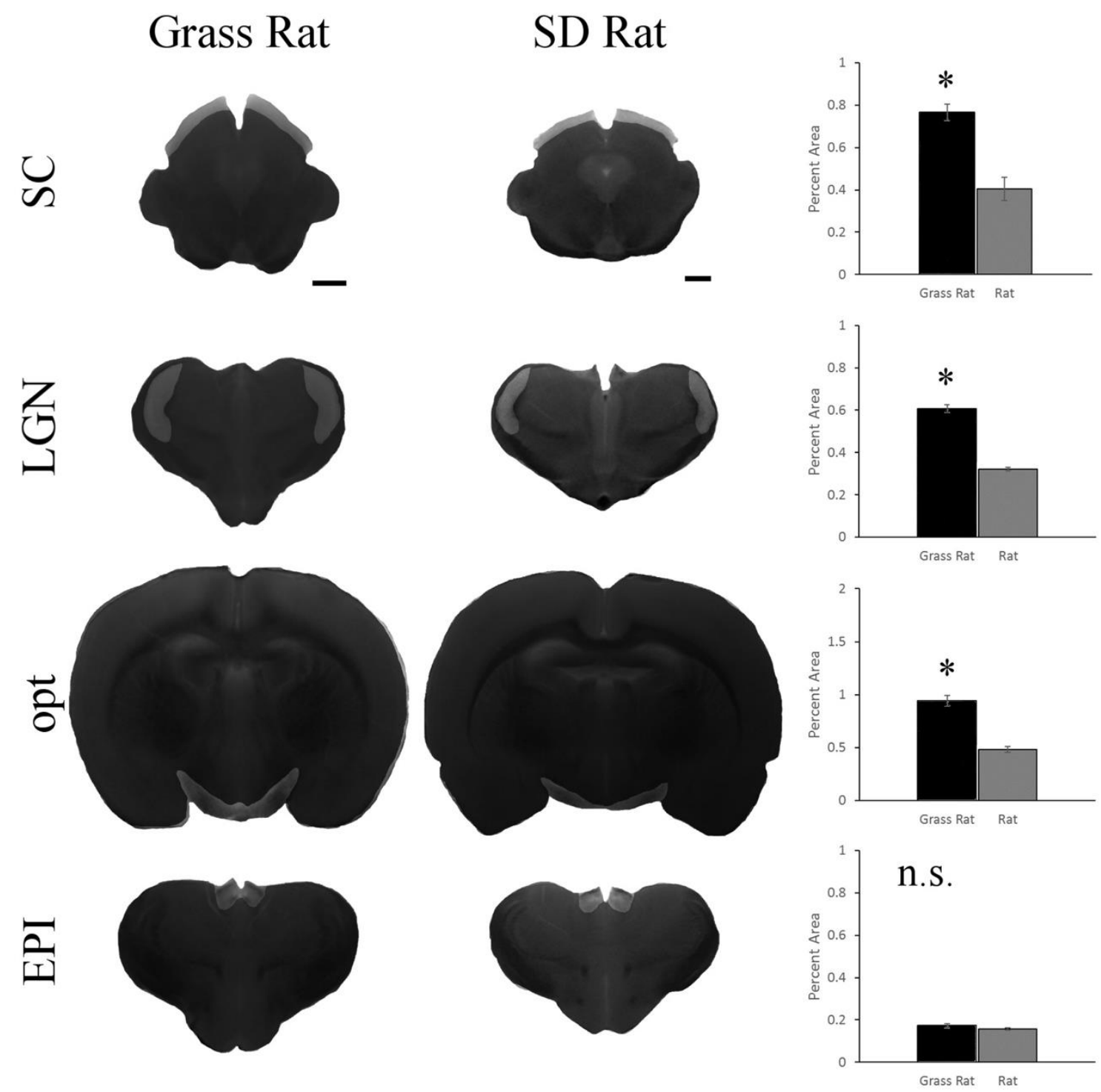

Figure 7. Volume analysis of visual system structures between species. The regions of interest within the visual system were delineated for both species (left columns). The grass rat had significantly larger relative SC, LGN and opt when compared to the SD rat (right column). However, the EPI between the two species were not different from one another. $*$ indicates $\mathrm{p}<0.05$. 


\section{Grass Rat}

\section{SD Rat}
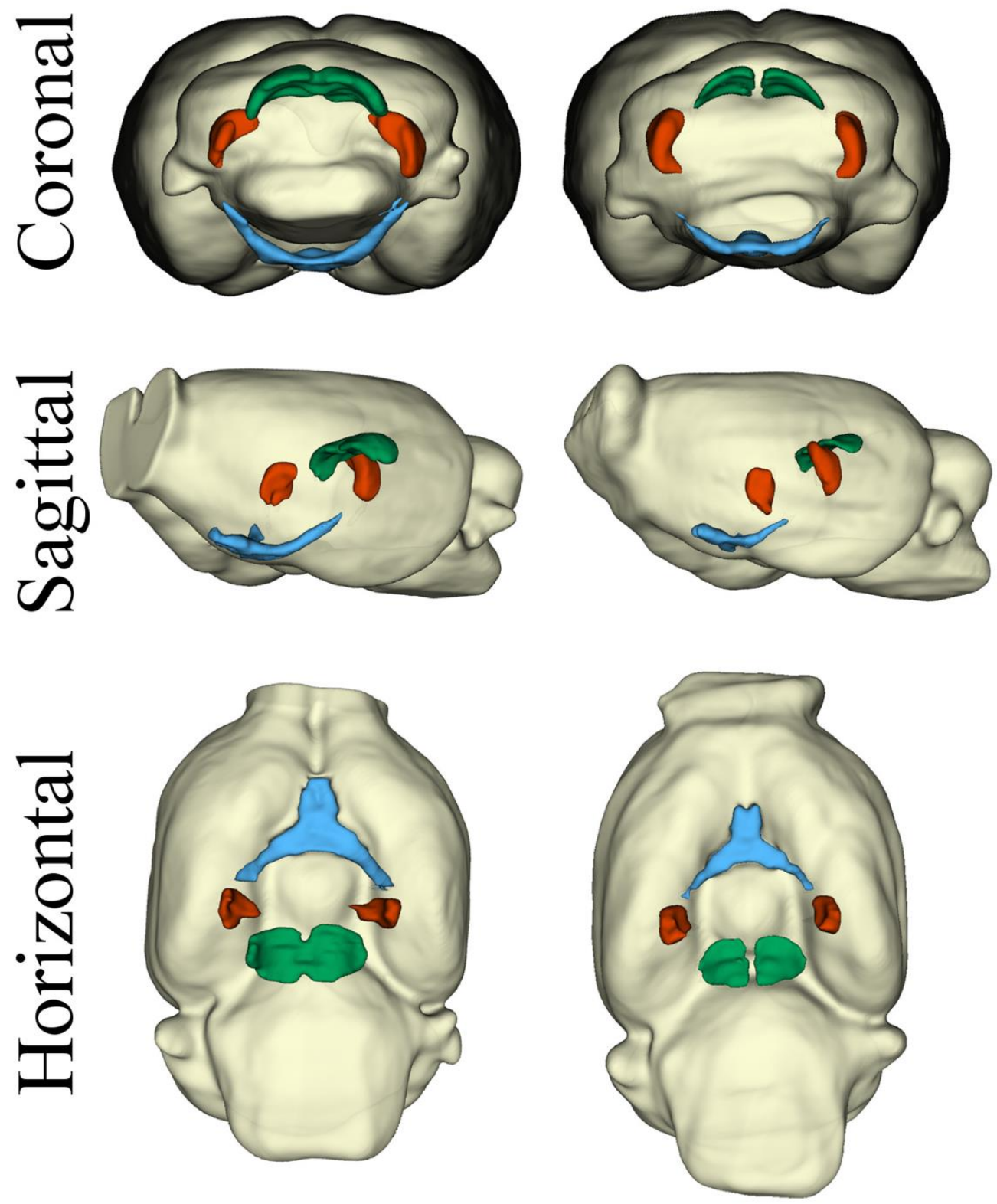

Figure 8. Three-dimensional volume rendering of visual regions in grass rat and SD rat from 3D MRI. Volumes for the superior colliculus (green), geniculate complex (red) and the optic nerve/tract (blue) are shown in three orientations. The brains of the two species are represented in identical proportions to demonstrate the relative size differences between visual structures. Grass rats have significantly larger regions for the three visual regions illustrated. 